\section{Emergencies in Obstetrics and Gynaecology}

by Lindsey Stevens with Anthony Kenney

Oxford University Press, 1994. £16.95. 265 pp.

ISBN 0-19-262051-7

Up to $5 \%$ of patients presenting to accident and emergency (A\&E) departments have a gynaecological or obstetric condition, yet many junior A\&E doctors have little or no experience in this field.

In her book, Emergencies in Obstetrics and Gynaecology, Lindsey Stevens provides a comprehensive overview of the initial diagnosis and management of acute gynaecological and obstetric problems, ranging from the most common, e.g. vaginal discharge and miscarriage, to the rare, e.g. trauma in pregnancy or rape. There are lists of key points at the start of each chapter and the text is supplemented with useful flow charts based on clinical findings to give differential diagnoses and management protocols.

The section of normal vaginal delivery would prove invaluable to the single-handed A\&E officer faced with a patient with imminent delivery of a concealed pregnancy and no obstetric unit on site!

Although legal and ethical issues are discussed in some depth, I was disappointed that there was no mention of the need for a chaperone during vaginal examination. Some important issues need clarification. It was initially suggested that vaginal examination could be performed for bleeding in pregnancy except in the third trimester (p. 11) but later a more appropriate time limit of 20 weeks' gestation was given. The units used throughout the book need to be consistent and it would be preferable for blood gases to be quoted in $\mathrm{KPa}$ throughout and blood sugars in $\mathrm{mmols} / \mathrm{L}$ instead of $\mathrm{mg} / \mathrm{dL}$.

I would have liked to have seen a section on 'resuscitation in pregnancy' and the section on pelvic inflammatory disease expanded to take into account unusual presentations of pelvic inflammatory disease, e.g. septic arthritis and perihepatitis.

The section reproduced from the BNF on prescribing in pregnancy is useful but may become out-dated. It would have be useful for drug interactions with the oral contraceptive pill to have been mentioned. both the A\&E officer and the junior doctor in obstetrics and gynaecology, yet is sufficiently compact that it can be read from cover to cover in a day. I found it hard to put down; but perhaps that was because I was reading it whilst on maternity leave!

\section{P. NASH}

Consultant, Accident and Emergency Department, Neath General Hospital, West Glamorgan, Wales

\section{Maxillofacial and Dental Emergencies by Hawkesford and Banks, Oxford University Press, 1994. 184 pp. ISBN 0192619977}

Maxillofacial and dental emergencies frequently present in the accident and emergency (A\&E) department. Anyone who requires a basic grounding in presentation and management need to look no further than this excellent handbook. The primary focus of this book is dental and maxillofacial trauma and the authors quite rightly present their material in the context of increasing violent crime.

There are numerous high quality line diagrams, radiographs and clinical photographs and many useful check lists. It is clear that the objective has been to produce a book of direct relevance to A\&E doctors and nurses, although there is also much here for senior undergraduates.

Acute conditions of the oral mucosa and salivary glands are not neglected and the text is supplemented by informative colour photographs. As befits a practical clinical handbook like this, there are suggestions for further reading rather than comprehensive lists of references and there are also referral guidelines.

This book is an important component of the Oxford University Press series of handbooks in Emergency Medicine. They are right size for carrying around in a white coat and this latest addition is no exception.

\section{J.P. SHEPHERD}

Professor of Oral and Maxillofacial Surgery University of Wales College of Medicine, Cardiff Wales 